$\begin{array}{ll}\text { Research Square } & \begin{array}{l}\text { Preprints are preliminary reports that have not undergone peer review. } \\ \text { They should not be considered conclusive, used to inform clinical practice, } \\ \text { or referenced by the media as validated information. }\end{array}\end{array}$

\title{
Optimization of Processing Technology Based on Chemical Composition Difference and Anti-Fibrotic Activity
}

\section{Chuan Ding}

Zhejiang Chinese Medical University

\section{Peng Xu}

Zhejiang Chinese Medical University Affiliated Third Hospital

\section{Yan Ning}

Zhejiang Chinese Medical University

QiYuan Shan

Zhejiang Chinese Medical University

\section{Minjie Niu}

Nanjing University of Chinese Medicine

\section{Hao Cai}

Nanjing University of Chinese Medicine

Xin Han ( $\nabla$ hanxin0906@yahoo.com )

Zhejiang Chinese Medical University https://orcid.org/0000-0002-1725-7940

\section{Gang Cao}

Zhejiang Chinese Medical University

\section{Research}

Keywords: Cornus officinalis, fibrosis, wine steamed, processing technology

Posted Date: November 2nd, 2021

DOI: https://doi.org/10.21203/rs.3.rs-1030612/v1

License: (c) (i) This work is licensed under a Creative Commons Attribution 4.0 International License. Read Full License 


\section{Abstract \\ Background}

Cornus officinalis, a kind of edible herbal medicine, has been widely used in liver and kidney protection due to its anti-inflammatory, anti-tumor, and anti-oxidant activities, which can be enhanced with winesteamed (WS) processing.

\section{Methods}

Based on the activations of HSC-T6 and HK-2, our study used single-factor plus orthogonal design to investigate the anti-fibrosis of $C$. officinalis processed with steamed (S), high-pressure steamed (HPS), WS, high-pressure wine-steamed (HPWS), wine-dipped (WD), and wine-fried (WF). The chemical constituents in processed $C$. officinalis with higher anti-fibrotic activities were detected by UPLC-Q-TOFMS/MS.

\section{Results}

Results showed that $C$. officinalis with HPWS significantly inhibited the activations of HSC-T6 and HK-2. Moreover, compounds in $C$. officinalis with HPWS were obtained via UHPLC-Q-TOF-MS/MS, indicating that 27 components were changed compared with raw $C$. officinalis.

\section{Conclusions}

These results demonstrated that HPWS is the optimal processing technology for anti-fibrosis of $C$. officinalis.

\section{Introduction}

Cornus officinalis, commonly used in traditional Chinese medicine (TCM), is the dry mature fruit of $C$. officinalis Sieb. et Zucc (Cornaceae) and is redefined as a class of herb and edible plant [1]. C. officinalis, with a mild warm nature, belongs to the meridians of the liver and kidney according to TCM theory; thus, it is commonly used in the prevention and treatment of liver and kidney diseases [2]. Moreover, it can be found in foodstuff, such as medicinal dishes and healthcare products and drinks due to its various pharmacological activities, including anti-inflammatory, antioxidant and anti-apoptotic [3]. To date, about 305 components have been isolated and identified from $C$. officinalis, including iridoids, alkaloids, polysaccharides, flavones, organic acid, essential oils and terpenoids [1]. Among these compounds, loganin and morroniside-active ingredients isolated from $C$. officinalis-have been shown to alleviate osteoarthritis in mice by inhibiting pyroptosis and NF-kappaB activity [4-5]. Furthermore, morroniside could ameliorate neuropathic pain through the regulation of glucagon-like peptide-1 (GLP-1) receptors. 5- 
hydroxymethylfurfural (5-HMF), which are mainly isolated from processed $C$. officinalis, could prevent human umbilical vein endothelial cells (HUVECs) from oxidative stress induced by glucose [6]. However, the compounds in $C$. officinalis and its pharmacological activity may change with processing. In traditional crafts of China, $C$. officinalis is often processed using fried, steamed, wined, fated, salted processing techniques, among others, to achieve different clinical effects. For example, wined $C$. officinalis is most commonly used in clinical preparations, such as the Liuwei Dihuang pills in China, which has better effects in nourishing the liver and kidney compared to raw $C$. officinalis. However, there is no unified requirement for steaming and braising times and wine amount, all of which could change in the compositions and activities of $C$. officinalis. Therefore, the present study aimed to optimize the processing technology of $C$. officinalis through the single-factor method and an orthogonal experiment design based on chemical composition difference and the anti-fibrotic activity of $C$. officinalis.

Tissue fibrosis, defined as the excessive deposition of extracellular matrix (ECM), is the outcome of chronic tissue damage, leading to the formation of scar tissue and even organ dysfunction and failure [7]. ECM is mainly derived from proliferative and fibrogenic myofibroblasts, which are fibroblast-like cells that possess contractile properties [8, 9]. Activated hepatic stellate cells (HSC-T6) are the main source of myofibroblasts, which serve as a key driver of hepatic fibrosis in liver injuries [9]. In normal liver, HSC-T6 are in quiescent state and are mainly used to store vitamin A [10]. In comparison, during liver injury, quiescent HSC-T6 are activated and indicated by the high expression of alpha smooth muscle actin (aSMA) and excessive deposition of ECM [11]. Similar to HSC-T6, human proximal tubular epithelial cell (HK-2) cells play a key role in tubulointerstitial fibrosis (TIF), which is the final result of chronic kidney disease and is closely related to the degeneration of renal functions [12]. Moreover, epithelial mesenchymal transition (EMT) is the main pathogenesis of renal fibrosis and can transform differentiated epithelial cells into myofibroblasts that are also characterized by the high expression of aSMA $[13,14]$. During fibrogenesis, EMT may be driven by various pro-fibrotic growth factors, among which transforming growth factor $\beta$ (TGF- $\beta$ ) is the most important factor [15]. Apart from these, TGF- $\beta$ is regarded as a common vital switch for fibrosis in tissue or organs in response to chronic injuries [16].

In summary, we will evaluate the effects of $C$. officinalis on anti-fibrosis via the expression of a-SMA in activated HSC-T6 and differentiated HK-2 cells induced by TGF- $\beta$, with the goal of exploring the protective effect of $C$. officinalis on the liver and kidney, and finally selecting the processing technology with higher anti-fibrotic activity. Moreover, changes of chemical constituents in processed $C$. officinalis will be detected by ultra-high performance liquid chromatography coupled with hybrid triple quadrupole time-offlight mass spectrometry (UHPLC-Q-TOF-MS/MS).

\section{Materials And Methods}

\subsection{Plant materials and processing technology}

The samples of $C$. officinalis were selected from the PanAn City of Zhejiang Province and identified by Professor Jianwei Chen of the Department of Chinese Medicine Identification, School of Pharmacy, 
Nanjing University of Chinese Medicine. According to single-factor experiments, $C$. officinalis samples were steamed at different times $(1,2,4,6$, and $8 \mathrm{~h})$ at different temperatures $\left(100^{\circ} \mathrm{C}, 105^{\circ} \mathrm{C}, 110^{\circ} \mathrm{C}, 115^{\circ} \mathrm{C}\right.$, $120^{\circ} \mathrm{C}$ and $125^{\circ} \mathrm{C}$ ) and then dried at $60^{\circ} \mathrm{C}$, respectively. Next, the samples were braised at different times $(0.5,1,2$, and $4 \mathrm{~h})$ with different dosages of rice wine $(\mathrm{w} / \mathrm{w})(15 \%, 20 \%, 25 \%, 30 \%$, and $40 \%)$, respectively. These samples were then steamed again for $1 \mathrm{~h}$ at $105^{\circ} \mathrm{C}$ and then finally dried at $60^{\circ} \mathrm{C}$. The orthogonal experimental design was based on the results of single-factor experiments, as follows: $C$. officinalis samples were processed with three factors (steamed times, steamed temperatures, braised times) and at different dosages of rice wine in line with $L^{9}\left(3^{4}\right)$ design orthogonal table shown in $S 1, S 3$, and S5, respectively.

\subsection{Extraction preparation}

Both raw and processed $C$. officinalis were crushed and sifted through 16-mesh screen. The powder $(5 \mathrm{~g})$ was refluxed with $90 \%$ ethanol $(50 \mathrm{~mL})$ at a water bath $\left(100^{\circ} \mathrm{C}\right)$ twice and filtered with four layers of gauze. The filtrates were collected and combined. Next, the filtrates were transformed into freeze-dried powder through vacuum concentration and lyophilization.

\subsection{UHPLC-Q-TOF-MS/MS analysis}

The LC-MS/MS analysis were performed using an UHPLC (Shimadzu LC-30AD, Japan) coupled with a Triple TOF 5600 Plus System (AB Sciex, USA). The following parameters were followed: column temperature was $30^{\circ} \mathrm{C}$, mobile phase was acetonitrile $(B)$ and $0.1 \%$ formic acid in water $(A)$, flow rate was $0.3 \mathrm{~mL} / \mathrm{min}$, and the injection volume was $3 \mu \mathrm{L}$. The gradient elution procedure was as follows: $0-3.0$ min, 5-20\% B; 3.0-7.0 min, 20-80\% B; 7.0-30 min, 80-90\% B; 30-32 min, 90-5\% B; 30-32 min, B was maintained at $5 \%$. For HILIC separation, the samples were analyzed with a $2.1 \mathrm{~mm} \times 100 \mathrm{~mm}$ ZORBAX Extend-C18 $1.8 \mu \mathrm{m}$ column (Agilent, USA). The conditions in both electrospray ionization (ESI) positive and negative modes were set as follows: the ion source temperature was $550^{\circ} \mathrm{C}$, lonSpray Voltage was 5500-5500 V, auxiliary spray gas was nitrogen, lon Source Gas1 (Gas1) was 55 psi, lon Source Gas2 (Gas2) was 55 psi, curtain gas (CUR) was 35 psi, declustering potential (DP) was $60 \mathrm{~V}$, and collision energy was 30V. The scanned ranges of TOF-MS and TOF-MS/MS were 100-2000 and 50-1000 Da, respectively.

\subsection{Cell culture and treatment}

HSC-T6 and HK-2 cells were cultured in Dulbecco's modified Eagle medium (DMEM) (Abcam, UK) and Dulbecco's Modified Eagle Media DMEM/F12 (Abcam, UK), respectively, supplemented with $100 \mathrm{mg} / \mathrm{mL}$ streptomycin, $100 \mathrm{U} / \mathrm{mL}$ penicillin, and $10 \%$ fetal bovine serum (FBS) (Abcam, UK) under $5 \% \mathrm{CO} 2$ at $37^{\circ} \mathrm{C}$. When establishing liver fibrosis in vitro, the HSC-T6 and HK-2 cells were cultured in six-well plates and treated with TGF- $\beta$ (PeproTech, USA), respectively. To detect the effects of $C$. officinalis on anti-fibrosis, cells were treated with extracts derived from raw and processed $C$. officinalis.

\subsection{Western blot analysis}


Cells were lysed using RIPA buffer supplemented with $1 \%$ phenylmethanesulfonyl fluoride (PMSF) (Solarbio, Beijing, China) and then quantified by BCA protein assay kit (Beyotime, Shanghai, China). Equivalent protein samples were separate by sodium dodecyl sulfate-polyacrylamid gel electrophoresis (SDS-PAGE) and then transferred to polyvinylidene fluoride (PVDF) membranes (GE, Freiburg, Germany). The membranes were blocked with $5 \%$ skim milk for $1 \mathrm{~h}$ at room temperature and incubated with antialpha smooth muscle actin (a-SMA) antibody (Abcam, UK) overnight at $4^{\circ} \mathrm{C}$. The membranes were incubated with horse radish peroxidase (HRP)-conjugated secondary antibodies for $1 \mathrm{~h}$ at room temperature and visualized using ECL Reagent (Beyotime, Shanghai, China). Finally, the membranes were stripped and probed with GAPDH that a loading control. The intensities of bands were quantified by Quantity One software (Bio-Rad, Hercules, CA, USA).

\subsection{Immunocytochemistry}

HSC-T6 cells were cultured in six-well plates and fixed with 4\% paraformaldehyde, permeabilized in $0.1 \%$ Triton X-100, and then incubated with rabbit polyclonal anti-a-SMA (1:100 dilution) (Abcam, UK) at $4^{\circ} \mathrm{C}$ overnight. The cells were then incubated with goat anti-rabbit IgG H\&L (Alexa Fluor® 488, 1:200 dilution) after which cell nuclei were stained with DAPI (Beyotime, Shanghai, China). Finally, the images were observed and analyzed with a Nikon TI-E fluorescence microscope.

\subsection{Statistical analysis}

The data in the experiments were present as mean \pm SD. The comparison of the results was evaluated by GraphPad Prism program (Graphpad Software, Inc., San Diego, CA, USA) with one-way analysis of variance (ANOVA) and Tukey's multiple comparison tests. Statistical significance between groups was considered with a p-value less than 0.05. Difference analyses were detected by MarkerView ${ }^{\mathrm{TM}}$ and $\mathrm{T}$ Test. The original data were imported into MarkerView ${ }^{\mathrm{TM}}$ and statistical analysis via T Test. The t-test was employed to identify significant differences among processed products. Here, $P<0.05$ was considered a significant difference, and a t-value greater than 0 indicated an increase in ingredient contents.

\section{Results}

\subsection{Evaluation of raw $C$. officinalis on anti-fibrosis}

The cytotoxicity of $C$. officinalis on HSC-T6 and HK-2 cells were evaluation by CCK8-a widely used method in the detection of cytotoxicity and drug sensitivity. In our study, HSC-T6 and HK-2 cells were treated with different doses of $C$. officinalis $(0-10 \mathrm{mg} / \mathrm{mL})$ for 24,48 , and $72 \mathrm{~h}$. Results showed that the sample at a dose range of $0-1 \mathrm{mg} / \mathrm{mL}$ had little cytotoxicity on HSC-T6, while the sample at a dose of 10 $\mathrm{mg} / \mathrm{mL}$ significantly inhibited the survival of cells compared with the normal sample at 24,48 , and $72 \mathrm{~h}$. For HK-2 cells, the minimal cytotoxicity of $C$. officinalis at a dose range of $0-1 \mathrm{mg} / \mathrm{mL}$ was observed only at $48 \mathrm{~h}$ and not at 24 and $72 \mathrm{~h}$ (Figures $1 \mathrm{~A}, \mathrm{~B}$, and C, respectively). Therefore, we chose the administration time at $48 \mathrm{~h}$ for the follow-up experiments. 
To establish a preferable fibrosis model in vitro, HSC-T6 and HK-2 were treated with different concentrations of TGF- $\beta$ at different times. The results showed that TGF- $\beta(1,5,10$, and $20 \mathrm{ng} / \mathrm{mL})$ activated HSC-T6 and HK-2 cells at different times $(24,48$, and $72 \mathrm{~h})$, which manifested by the higher expressions of a-SMA compared to the normal group (Figures 1D and E, respectively). Upon comparing the results, TGF- $\beta(10 \mathrm{ng} / \mathrm{mL})$ at $48 \mathrm{~h}$ was used to activate the cells. Moreover, results in Figure 1D showed that $C$. officinalis at different doses $(0.25,0.5$, and $1 \mathrm{mg} / \mathrm{mL})$ inhibited the expression of a-SMA in HSC-T6 and HK-2 cells induced by TGF- $\beta$, with the best effect identified at a dose of $1 \mathrm{mg} / \mathrm{mL}$ (Figure 1F).

\subsection{Effects of processed $C$. officinalis on anti-fibrosis changes with univariate elements}

The processing methods of $C$. officinalis found in the Pharmacopoeia of the People's Republic of China (ChP) including wine steamed (WS) and wine braised (WB) processing, among others. To identify and optimize the processing technology with the best anti-fibrosis activity, single-factor experiment was used in the present study. The results of the present study showed that $C$. officinalis steamed at different times $(1,2,4,6$, and $8 \mathrm{~h})$ or temperatures $\left(100^{\circ} \mathrm{C}, 105^{\circ} \mathrm{C}, 110^{\circ} \mathrm{C}, 115^{\circ} \mathrm{C}, 120^{\circ} \mathrm{C}\right.$ and $\left.125^{\circ} \mathrm{C}\right)$ inhibited the expression of a-SMA both in HSC-T6 and HK-2 cells induced by TGF- $\beta$. Among these, the anti-liver fibrosis activity of $C$. officinalis steamed for $4 \mathrm{~h}$ at $100^{\circ} \mathrm{C}$ was the best, while that steamed at $1 \mathrm{~h}$ at a temperature of $105^{\circ} \mathrm{C}$ had the best anti-fibrosis effects on HK-2 cells (Figures $2 \mathrm{~A}$ and $\mathrm{B}$, respectively). Furthermore, braised $C$. officinalis for $0.5,1,2$ and $4 \mathrm{~h}$ inhibited TGF- $\beta$-induced over-expression of a-SMA in HSC-T6 and HK-2 cells, especially at $1 \mathrm{~h}$ in HSC-T6 and $4 \mathrm{~h}$ in HK cells (Figure 2C). C. officinalis processed with different dosages of rice wine $(\mathrm{w} / \mathrm{w})$ also showed different degrees of anti-fibrosis activity, especially $15 \%$ in HSC-T6 and 25\% in HK-2 cells (Figure 2D).

The results showed that the anti-fibrosis effect of $C$. officinalis is negatively correlated with the expression of a-SMA. Thus, the data were non-negatively analyzed, as follows: $X i j=m a x ~(X 1 j, X 2 j, \ldots . . . X n j)-$ $X i j / \max (X 1 j, X 2 j, \ldots . . . X n j)-\min (X 1 j, X 2 j, \ldots \ldots . . X n j)+1 ; i=1 \otimes 2, \ldots . . n, j=1,2 \ldots . . n)$. Moreover, the expressions of $a-$ SMA in HSC-T6 and HK-2 cells were used as indicators for comprehensive scoring, each with a weight of 0.5. The composite score was calculated using the following formula: Xij (HK-2) *0.5+Xij (HSC-T6) *0.5. The results showed that $C$. officinalis processed with the following parameters: steaming time of $1 \mathrm{~h}$, steaming temperatures of $105^{\circ} \mathrm{C}$ and $125^{\circ} \mathrm{C}$, braising times of 0.5 and $4 \mathrm{~h}$, and rice wine dosage $(\mathrm{w} / \mathrm{w})$ at $25 \%$, respectively, had the best inhibitory effects on activated HSC-T6 and HK-2.

\subsection{Optimization of the processing technology with orthogonal test}

In accordance with the terms of the single-factor experiments, the processing parameters were optimized with an orthogonal $\mathrm{L}^{9}\left(3^{4}\right)$ test design. Our results showed that $C$. officinalis processed at three levels of two factors (steaming times and steaming temperatures), as shown in Table $\mathrm{S} 1$, inhibited the expressions of a-SMA in HSC-T6 and HK-2 induced by TGF- $\beta$ (Figure 3A). The composite score of the anti-fibrosis effects was calculated with non-negative analysis, and the results indicated that the maximum was 1.92 
(Table S1). Furthermore, in selecting the better processing term, the values of $\mathrm{K}$ and $\mathrm{R}$ are shown in Table $\mathrm{S} 1$, and the variance analysis results are shown in Table S2. These results showed that $C$. officinalis processed for $1 \mathrm{~h}$ at $125^{\circ} \mathrm{C}$ (known as high-pressure steaming (HPS) showed optimum anti-fibrosis effect. The craft conditions of WS $C$. officinalis were also optimized with an orthogonal design (Table S3), and its anti-fibrosis effects on both HSC-T6 and HK-2 were evaluated via the over-expression of a-SMA induced by TGF- $\beta$ (Figure 3B). The results indicated that WS $C$. officinalis with different inhibited the activation of myoblasts, which was mainly manifested by the decrease of a-SMA expressions (Figure 3B). Combined with the results of variance analysis (Table S4) and the values of $\mathrm{K}$ and $\mathrm{R}$ in Table S3, the optimal processing term with anti-fibrosis effects were as follows: steaming time of $2 \mathrm{~h}$, braising time of $0.5 \mathrm{~h}$, and rice wine dosage $(\mathrm{w} / \mathrm{w})$ of $30 \%$. Similarly, the optimal parameters of high-pressure wine steamed (HPWS) C. officinalis were identified (steaming for $1 \mathrm{~h}$ at $115^{\circ} \mathrm{C}$, braised time of $1 \mathrm{~h}$, and rice wine dosage $(\mathrm{w} / \mathrm{w})$ at $25 \%$ ), all of which were optimized with orthogonal design (Table S5), variance analysis (Table S6), and the expression of a-SMA (Figure 3C).

\subsection{Validation of processed $C$. officinalis in terms of anti- fibrosis effects}

The optimal processing technologies of processed $C$. officinalis were determined by single-factor and orthogonal experiments. According to the terms of HPWS C. officinalis and the stipulation in Chp, winedipped (WD) and wine-fried (WF) C. officinalis samples were prepared. The expressions of a-SMA were detected by Western blot to verify the anti-fibrosis activity of all processed $C$. officinalis. The results showed that both raw and processed $C$. officinalis inhibited the expressions of a-SMA induced by TGF- $\beta$ in both HSC-T6 and HK-2, especially $C$. officinalis processed with HPWS (Figure 4A). Then, immunofluorescence assay for a-SMA in HSC-T6 was conducted to confirm the anti-fibrosis effect enhanced by $C$. officinalis processed with HPWS. The results also showed that $C$. officinalis inhibited the positive expression of a-SMA (in green) induced by TGF- $\beta$, especially the sample processed with HPWS (Figure 4B).

\subsection{Ingredient identification of $C$. officinalis processed with HPWS}

The analysis of the compounds in both raw and HPWS samples were identified by ESI positive and negative modes (Figure 5). The chemical name, molecular mass, molecular formula, and molecular structure of components in $C$. officinalis were retrieved and download from the database, after which the accurate mass-to-charge ratio of plasma morphology were calculated in both ESI positive and negative modes. The raw data were imported into the PeakView ${ }^{\mathrm{TM}}$ software. All the chemical components were encoded and a new session was established under the XIC Manager template. Then, first-level data matching was conducted with reference standards, standard mass spectrometric database, and literature according to $\mathrm{m} / \mathrm{z}$. The chromatographic peak with the retention time error within $0.2 \mathrm{~min}$ and the $\mathrm{m} / \mathrm{z}$ error within $10 \mathrm{ppm}$ was identified as a unified compound. Further, the identification validation and 
chromatographic peak attribution were based on molecular structures and secondary fragments of compounds.

As shown in Table 1, 27 components in $C$. officinalis were changed in the HPWS condition, including flavonoids, iridoid glycosides, and organic acids. Moreover, 5-HMF was a typical emerging compound after $C$. officinalis was processed with HPWS. We take quercetin as an example to illustrate the identification procedure: $[\mathrm{M}-\mathrm{H}]^{-}$of peak 11 was 301.0354 , formula was calculated as $\mathrm{C}_{15} \mathrm{H}_{10} \mathrm{O}_{7}$ with Mass (Da) 302.0427, the main secondary fragment was 121.0305, and neutral loss was 180.0019. After consulting the literature [17] and comparing the results with the standard, we confirmed that the compound is quercetin.

\section{Discussion}

In TCM, C. officinalis is often used in replenishing the liver and kidney due to its tonic effect [1]. Furthermore, modern pharmacology indicates that $C$. officinalis shows low toxicity on cells only at high concentrations [18], as confirmed by the results of CCK8 in the present study. The present study also provides a theoretical basis for further research on liver- and kidney-related diseases. In liver and kidney fibrosis, persistent or dysregulated fibrogenic reactions may hamper regeneration and promote dysfunction [19], which could ultimately raise susceptibility to organ failure and death [20]. During these processes, TGF- $\beta$, which is a key cellular factor of fibrotic reactions, can contribute to a fibrogenic phenotype by activating fibroblasts cells [19], including HSC-T6 and HK-2 cells. Taking all these elements into account, the present study aimed to develop a therapeutic implementation of anti-TGF- $\beta$ approaches. The results of this study showed that $C$. officinalis downregulated the TGF- $\beta$-induced expression of $a-$ SMA by inhibiting the activation of fibroblast cells, thus providing a direction for us to optimize the processing technology of $C$. officinalis with higher anti-fibrotic activity.

Wined $C$. officinalis is a traditional and common processing method that is applied until now. However, different indexes of evaluation are accompanied by varying wine processing techniques. Thus, the current research aimed to explore the suitable wined technology of $C$. officinalis based on the anti-fibrosis synergistic effects produced by each technique. The results of univariate elements lay the foundation for subsequent orthogonal experiments. However, considering the time benefit, the term of orthogonal experiment was finally determined as follows: steaming times were 1,2 , and $3 \mathrm{~h}$; steaming temperatures were $105^{\circ} \mathrm{C}, 115^{\circ} \mathrm{C}$, and $125^{\circ} \mathrm{C}$; braising times were $0.5,1$, and $1.5 \mathrm{~h}$; and rice wine dosages $(\mathrm{w} / \mathrm{w})$ were $25 \%, 30 \%$, and $35 \%$. Due to the fact that steaming time, steaming temperature, braising time, and rice wine dosage are vital criteria in the processing of $C$. officinalis [21], processed samples using different technologies have varying antidiabetic effects [22]. Aside from this, the results of our study showed that the samples had different inhibitory effects on activated fibrosis cells. Therefore, optimization of processing criteria is the essential step in screening the technology with the optimal anti-fibrosis effect. Studies have shown that the orthogonal test design is a common method for the optimization of experimental conditions [23-24]. Thus, the current study used the orthogonal $\mathrm{L}^{9}\left(3^{4}\right)$ test design to detect 
the anti-fibrosis of $C$. officinalis processed with different factors for the single-factor experiments and obtained several kinds of samples via S, WS, and HPWS processing technologies.

Based on the single-factor and orthogonal tests, variance analysis, and the evaluation of anti-fibrosis activity, $C$. officinalis processed with HPWS showed better anti-fibrosis activity than other processed products. The pharmacological activities of $C$. officinalis are mainly due to various active components found in the $C$. officinalis [25], which are transformed during processing, thereby leading to differences in the anti-fibrosis activity of $C$. officinalis in liver. Therefore, exploring the composition changes in $C$. officinalis processed with HPWS can provide material basis for further clarifying the mechanism of $C$. officinalis with HPWS-enhanced anti-hepatic fibrosis.

To further excavate the differences in $C$. officinalis between raw and HPWS products, the data were standardized with SIMCA 14.1 (version, country) and analyzed via PLS-DA under supervised recognition mode. The results in Figure 5 present the PLS-DA score plot of $C$. officinalis before and after processing. As can be seen, the raw and HPWS processed $C$. officinalis products were obviously clustered into two categories, thereby indicating that the processing has changed the chemical composition of the sample.

The current results also showed that, after processing via HPWS, the chemical composition of $C$. officinalis changed qualitatively, including 5-HMF, linoelaidic acid, and quercetin. 5-HMF prevents L02 hepatocytes from injury induced by GaIN/TNF-a [26] and attenuates liver fibrosis by inhibiting oxidative stress in mice [27]. Furthermore, quercetin can protect the liver and kidney, as reported in another study [28]. Thus, the above research could reveal a chemical basis for the enhanced anti-fibrosis activity of $C$. officinalis processed by HPWS.

\section{Conclusion}

As a kind of medicine and edible herbal, $C$. officinalis has been shown to suppress liver and kidney fibrosis by inhibiting the activation of HSC-T6 and HK-2 cells. Studies have also shown that its antifibrosis activity can change with processing. Based on this information, our experiment used enhanced anti-fibrosis effects as the indicator to optimize the processing technology of $C$. officinalis through singlefactor and orthogonal tests. Finally, we identified the processing that can produce optimal anti-fibrosis activity, namely, $C$. officinalis with HPWS, whose chemical composition was identified by UHPLC-Q-TOFMS/MS analysis. The experimental result is a further step. The results of this study also provided material basis for further exploring the role of $C$. officinalis in liver and kidney protection.

\section{Abbreviations}

TCM, traditional Chinese medicine; 5-HMF, 5-hydroxymethylfurfural; HUVECs, human umbilical vein endothelial cells; ECM, extracellular matrix; a-SMA, alpha smooth muscle actin; HK-2, human proximal tubular epithelial cell; TIF, tubulointerstitial fibrosis; HSC-T6, hepatic stellate cells; EMT, epithelial mesenchymal transition; TGF- $\beta$, transforming growth factor $\beta$; UHPLC-Q-TOF-MS/MS, ultra-high 
performance liquid chromatography coupled with hybrid triple quadrupole time-of-flight mass spectrometry; ChP, Pharmacopoeia of the People's Republic of China; HPS, high-pressure steaming; HPWS, high-pressure wine steamed.

\section{Declarations}

Ethics approval and consent to participate

Not applicable

Consent to publish

Not applicable

\section{Availability of data and materials}

I agree to share my data and materials.

\section{Competing interests}

The authors declare no competing financial interest.

\section{Funding}

This work was financially supported by the National Natural Science Foundation of China (No.81973481, No.81922073 and No.8210142131), and the Traditional Chinese Medicine Key Scientific Research Fund Project of Zhejiang Province (No.2018ZY004).

\section{Authors' Contributions}

Gang Cao and Xin Han contributed to the design of the study, acquisition of data, and analysis and interpretation of the data. Chuan Ding, Peng Xu and Yan Ning contributed to the acquisition of data and analysis and interpretation of data. All authors participated in drafting or revising the manuscript and approved the final version of the manuscript for submission.

\section{Acknowledgements}

Not applicable

\section{References}

1. Huang J, Zhang Y, Dong L, Gao Q, Yin L, Quan H, et al. Ethnopharmacology, phytochemistry, and pharmacology of Cornus officinalis Sieb. et Zucc. J Ethnopharmacol. 2018; 213: 280-301. 
2. Gao X., Liu Y, An Z, Ni J. Active Components and Pharmacological Effects of Cornus officinalis: Literature Review. Front Pharmacol. 2021; 12: 633447.

3. Quah Y, Lee SJ, Lee EB, Birhanu BT, Ali MS, Abbas MA, et al. Cornus officinalis Ethanolic Extract with Potential Anti-Allergic, Anti-Inflammatory, and Antioxidant Activities. Nutrients. 2020; 12(11): 3317.

4. Hu J, Zhou J, Wu J, Chen Q, Du W, Fu F, et al. Loganin ameliorates cartilage degeneration and osteoarthritis development in an osteoarthritis mouse model through inhibition of NF-KB activity and pyroptosis in chondrocytes. J Ethnopharmacol. 2020; 247: 112261.

5. Yu H, Yao S, Zhou C, Fu F, Luo H, Du W, et al. Morroniside attenuates apoptosis and pyroptosis of chondrocytes and ameliorates osteoarthritic development by inhibiting NF-kappaB signaling. $J$ Ethnopharmacol. 2021; 266: 113447.

6. Cao G, Cai H, Cai B, Tu S, Effect of 5-hydroxymethylfurfural derived from processed Cornus officinalis on the prevention of high glucose-induced oxidative stress in human umbilical vein endothelial cells and its mechanism. Food Chem. 2013, 140 (1-2): 273-279.

7. Parola M, Pinzani M. Pathophysiology of Organ and Tissue Fibrosis. Mol Aspects Med. 2019; 65: 1.

8. Friedman S. L. Hepatic stellate cells: protean, multifunctional, and enigmatic cells of the liver. Physiol Rev. 2008; 88(1): 125-72.

9. Higashi T., Friedman SL, Hoshida Y. Hepatic stellate cells as key target in liver fibrosis. Adv Drug Deliv Rev. 2017; 121: 27-42.

10. Puche JE, Saiman Y, Friedman SL, Hepatic stellate cells and liver fibrosis, Compr Physiol. 2013; 3(4): 1473-1492.

11. Bataller R, Brenner DA. Liver fibrosis. J Clin Invest. 2005; 115(2): 209-218.

12. Zhang Y, Li K, Li Y, Zhao W, Wang L, Chen Z, et al. Profibrotic mechanisms of DPP8 and DPP9 highly expressed in the proximal renal tubule epithelial cells. Pharmacol Res. 2021; 105630.

13. Iwano M, Plieth D, Danoff TM, Xue C, Okada H, Neilson EG, Evidence that fibroblasts derive from epithelium during tissue fibrosis. J Clin Invest. 2002; 110(3): 341-350.

14. Loeffler I, Wolf G. Epithelial-to-Mesenchymal Transition in Diabetic Nephropathy: Fact or Fiction? Cells. 2015; 4(4): 631-652.

15. Meng XM, Tang PM, Li J, Lan HY. TGF- $\beta$ /Smad signaling in renal fibrosis. Front Physiol. 2015; 6: 82.

16. Weiskirchen R, Weiskirchen S, Tacke F. Organ and tissue fibrosis: Molecular signals, cellular mechanisms and translational implications. Mol Aspects Med. 2019; 65: 2-15.

17. Kao ST, Wang SD, Lin CC, Lin LJ. Jin Gui Shen Qi Wan, a traditional Chinese medicine, alleviated allergic airway hypersensitivity and inflammatory cell infiltration in a chronic asthma mouse model. J Ethnopharmacol. 2018; 227: 181-190.

18. Frangogiannis N. Transforming growth factor- $\beta$ in tissue fibrosis. J Exp Med. 2020; 217(3): e20190103.

19. Eming SA, Wynn TA, Martin P. Inflammation and metabolism in tissue repair and regeneration. Science. 2017; 56(6342): 1026-1030. 
20. Cao G, Cai H, Zhang Y, Cong X, Zhang C, Cai B. Identification of metabolites of crude and processed Fructus Corni in rats by microdialysis sampling coupled with electrospray ionization linear quadrupole ion trap mass spectrometry. J Pharm Biomed Anal. 2011; 56(1): 118-125.

21. Ma W, Wang KJ, Cheng CS, Yan GQ, Lu WL, Ge JF, et al. Bioactive compounds from Cornus officinalis fruits and their effects on diabetic nephropathy. J Ethnopharmacol. 2014; 153(3): 840-845.

22. Cai DJ, Shu Q, Xu BQ, Peng LM, He Y. Orthogonal test design for optimization of the extraction of flavonid from the Fructus Gardeniae. Biomed Environ Sci. 2011; 24(6): 688-693.

23. Wei L, Huang X, Huang Z, Zhou Z. Orthogonal test design for optimization of lipid accumulation and lipid property in Nannochloropsis oculata for biodiesel production. Bioresour Technol. 2013; 147: 534-538.

24. Dong Y, Feng ZL, Chen HB, Wang FS, Lu JH. Corni Fructus: a review of chemical constituents and pharmacological activities. Chin Med. 2018; 13: 34.

25. Cai H, Cao G, Cai B. Rapid simultaneous identification and determination of the multiple compounds in crude Fructus Corni and its processed products by HPLC-MS/MS with multiple reaction monitoring mode. Pharm Biol. 2013; 51(3): 273-278.

26. Jiang ZQ, Ma YX, Li MH, Zhan XQ, Zhang X, Wang MY. 5-Hydroxymethylfurfural protects against ER stress-induced apoptosis in GaIN/TNF-a-injured L02 hepatocytes through regulating the PERK-elF2a signaling pathway. Chin J Nat Med. 2015; 13(12): 896-905.

27. Han XY, Hu JN, Wang Z, Wei SN, Zheng SW, Wang YP, et al. 5-HMF Attenuates Liver Fibrosis in CCI4Plus-Alcohol-Induced Mice by Suppression of Oxidative Stress, J Nutr Sci Vitaminol (Tokyo), 2017, 63(1), 35-43.

28. Batiha GE, Beshbishy AM, Ikram M, Mulla ZS, El-Hack MEA, Taha AE, et al. The Pharmacological Activity, Biochemical Properties, and Pharmacokinetics of the Major Natural Polyphenolic Flavonoid: Quercetin. Foods. 2020; 9(3): 374.

\section{Tables}

Due to technical limitations, table 1 is only available as a download in the Supplemental Files section.

\section{Figures}



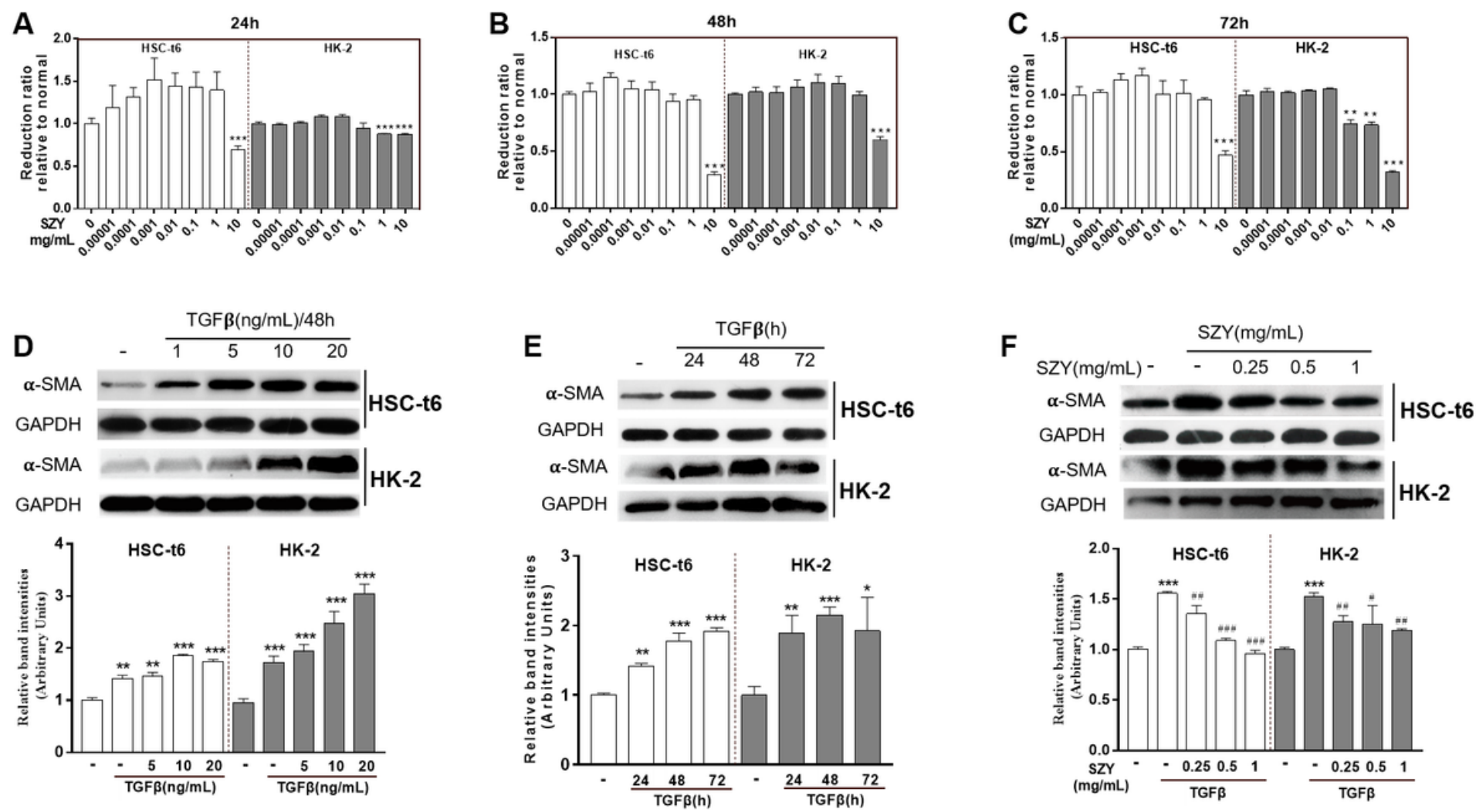

Figure 1

Pharmacodynamic evaluation of $\mathrm{C}$. officinalis. The cytotoxicity of $\mathrm{C}$. officinalis on HSC-T6 and HK2 cells for 24 (A), 48 (B), and $72 \mathrm{~h}$ (C). HSC-T6 and HK2 cells were activated by TGF- $\beta$ with different concentrations (D) and for different times $(E)$. (F) The effects of $C$. officinalis on activated HSC-T6and HK2 cells. Data are represented as means \pm SEM. ${ }^{*} P \leq 0.05$, ${ }^{\star * P} \leq 0.01$, and ${ }^{* \star * P} \leq 0.001$ vs. the control group; $\# \mathrm{P} \leq 0.05, \# P \leq 0.01$, and \#\#\# $\leq 0.001$ vs. the TGF- $\beta$ group. 

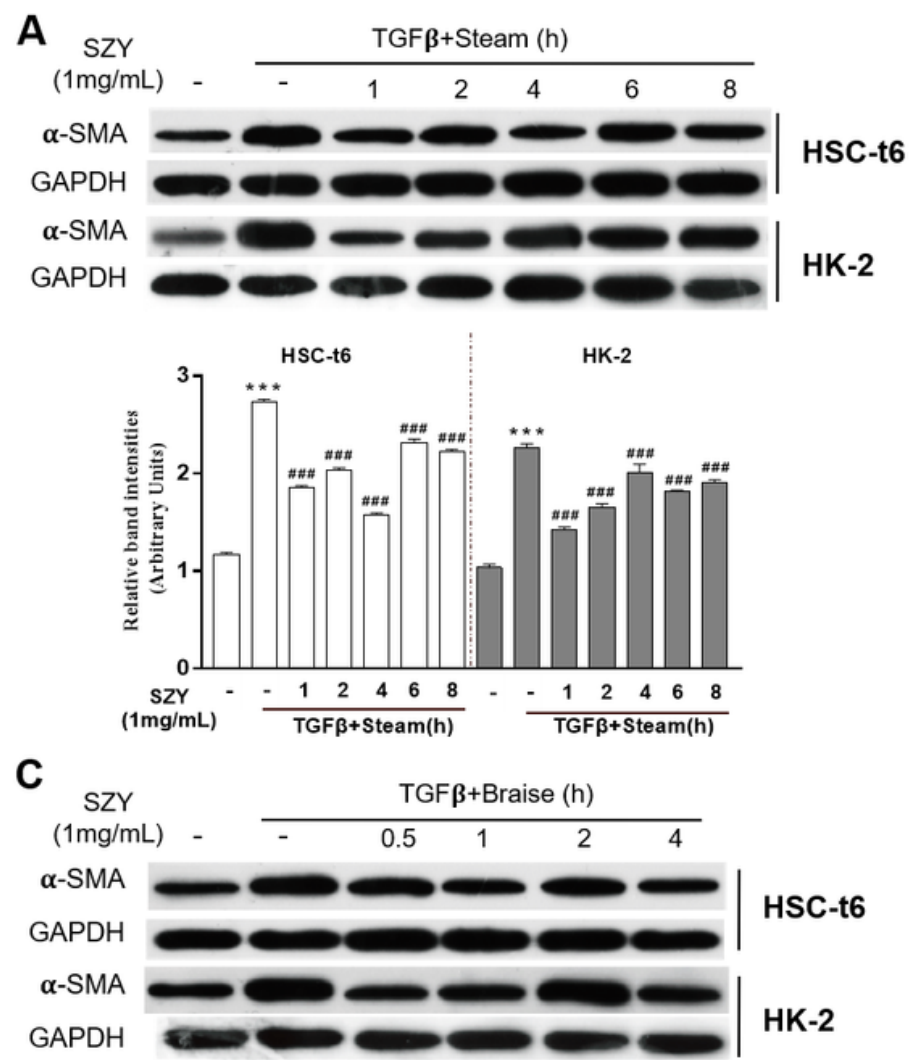

HSC-t6

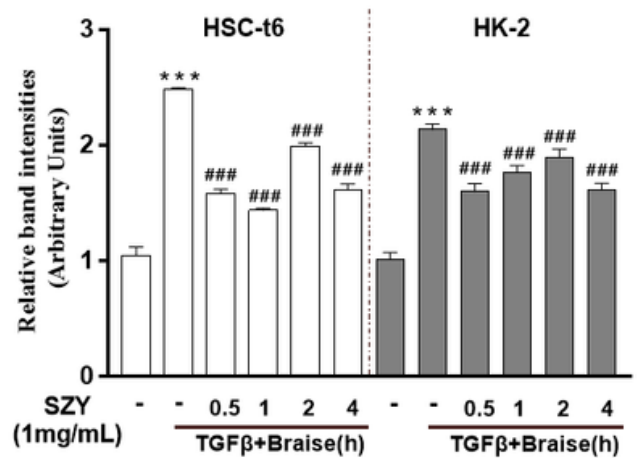

HK-2
B

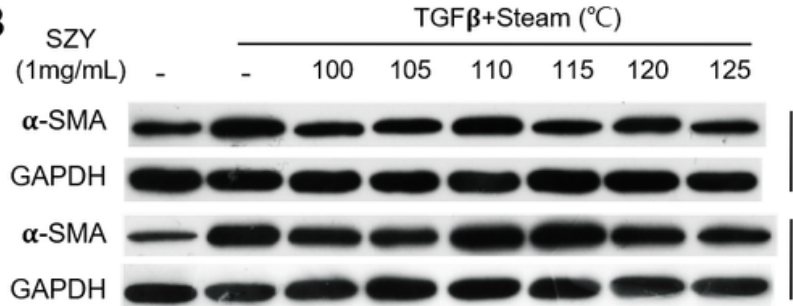

HSC-t6

HK-2

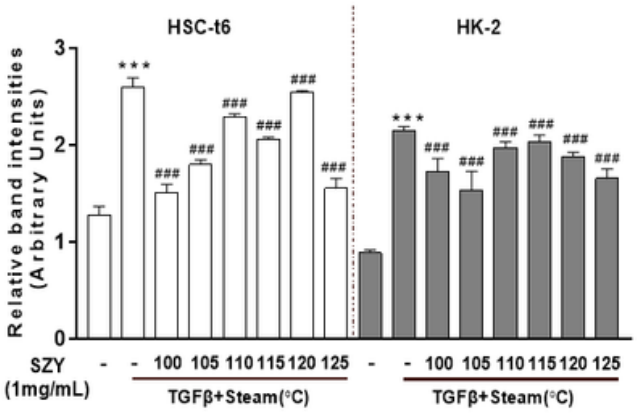

D

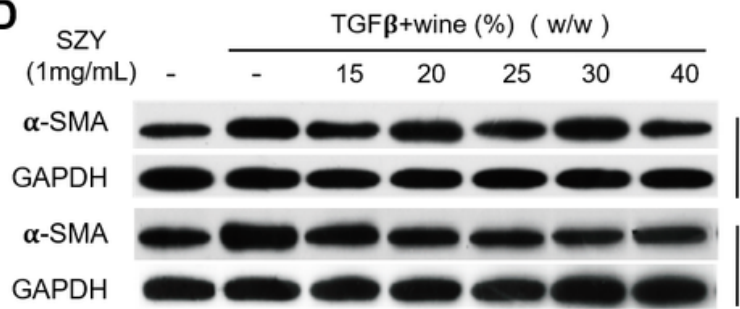

HSC-t6

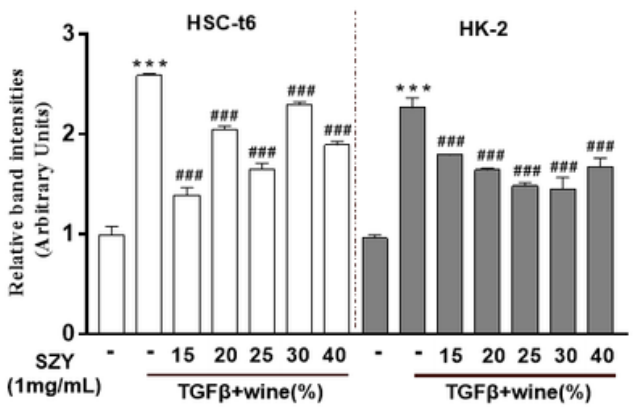

Figure 2

Effects of processed C. officinalis on anti-fibrosis changes with univariate elements. Anti-fibrosis activities of the $\mathrm{C}$. officinalis samples steamed at different times (A) and different temperatures (B) and braised at different times $(C)$ with different rice wine dosages $(w / W)(D)$. Data are represented as means \pm SEM. ${ }^{* \star *} \mathrm{P} \leq 0.001$ vs. the control group. \#\#\#P $<0.01$ vs. the TGF- $\beta$ group. 
A

SZY TGF $\beta+$ high pressure steamed

$(1 \mathrm{mg} / \mathrm{mL}) \quad-\quad-\quad \begin{array}{lllllllll}-1 & 2 & 3 & 4 & 5 & 6 & 7 & 8 & 9\end{array}$

$\alpha$-SMA $-\infty-\infty-\infty=0-\infty$

GAPDH

$\alpha-S M A-\infty-\infty-\infty-\infty=$

GAPDH Wm

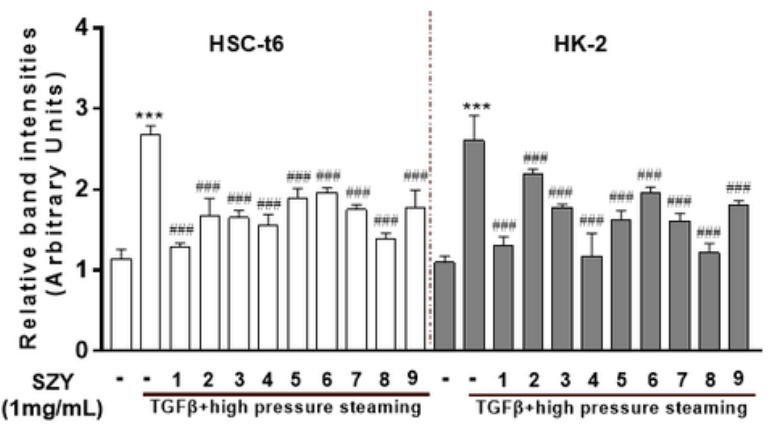

C

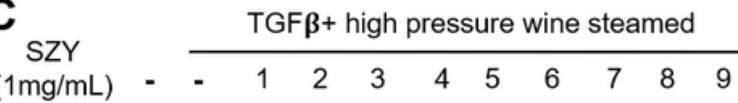

$\alpha$-SMA $-\infty-\infty-\infty-\infty$

GAPDH

$\alpha$-SMA - - - - - - - - -

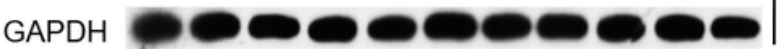

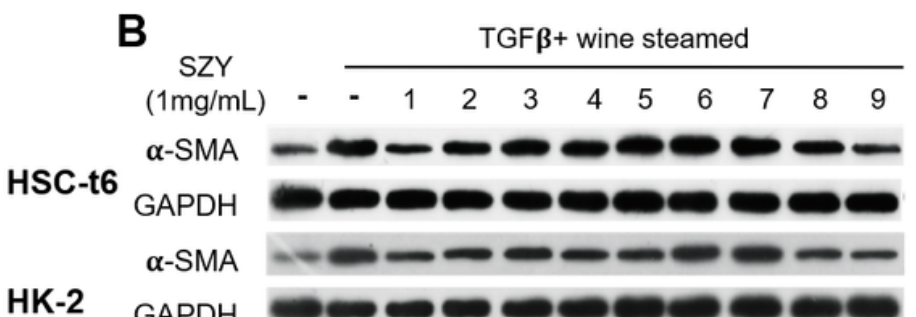

HSC-t6

HK-2

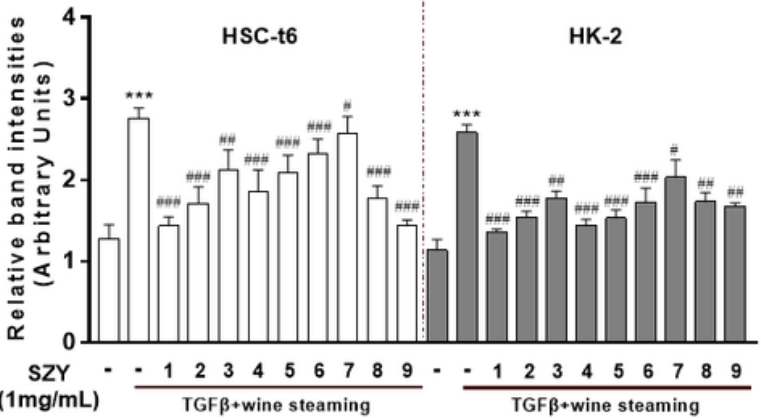

HSC-t6

HK-2

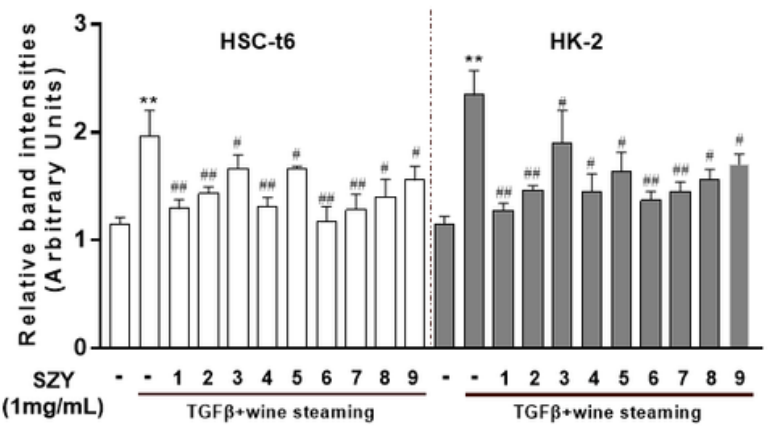

Figure 3

Optimization of the processing technology with orthogonal test. Anti-fibrosis activities of the $\mathrm{C}$. officinalis samples processed with HPS (A), WS (B), and HPWS (C) technologies. Data are represented as means \pm

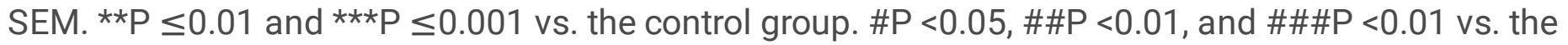
TGF- $\beta$ group. 
A
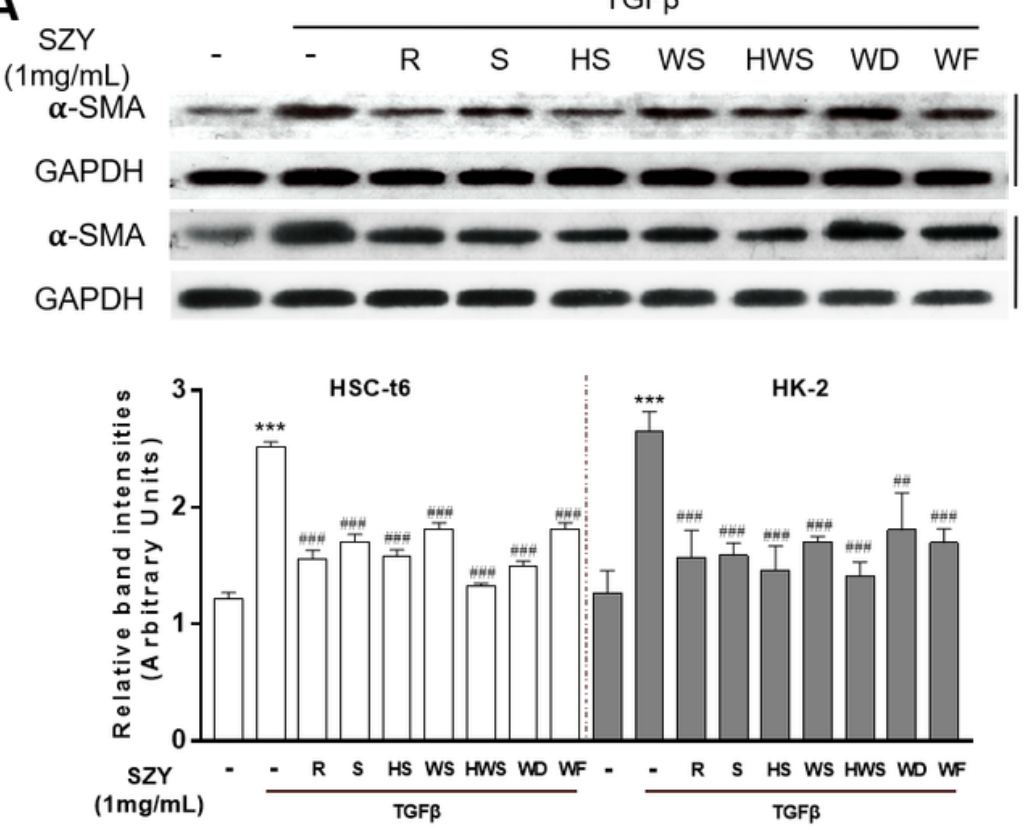

B

HSC-t6

HK-2
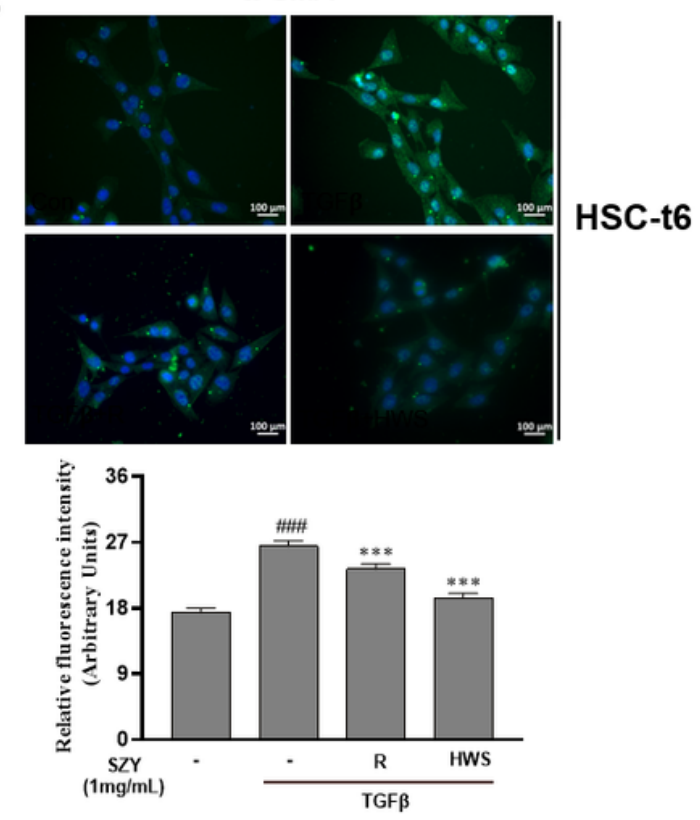

\section{Figure 4}

Validation of processed $\mathrm{C}$. officinalis in terms of anti-fibrosis activity. The anti-fibrosis activities of all processed $C$. officinalis samples were detected by Western blot analysis (A); the effects of

immunofluorescent staining of a-SMA in HSC-T6 treated with $\mathrm{C}$. officinalis processed with HPWS present in 400* magnification (B). Data are represented as means $\pm S E M . * P \leq 0.05, * * P \leq 0.01$, and ${ }^{* \star * P} \leq 0.001$ vs. the control group. \#P $<0.05$, \#\#P $<0.01$, and \#\#\#P<0.01 vs. the TGF- $\beta$ group. 

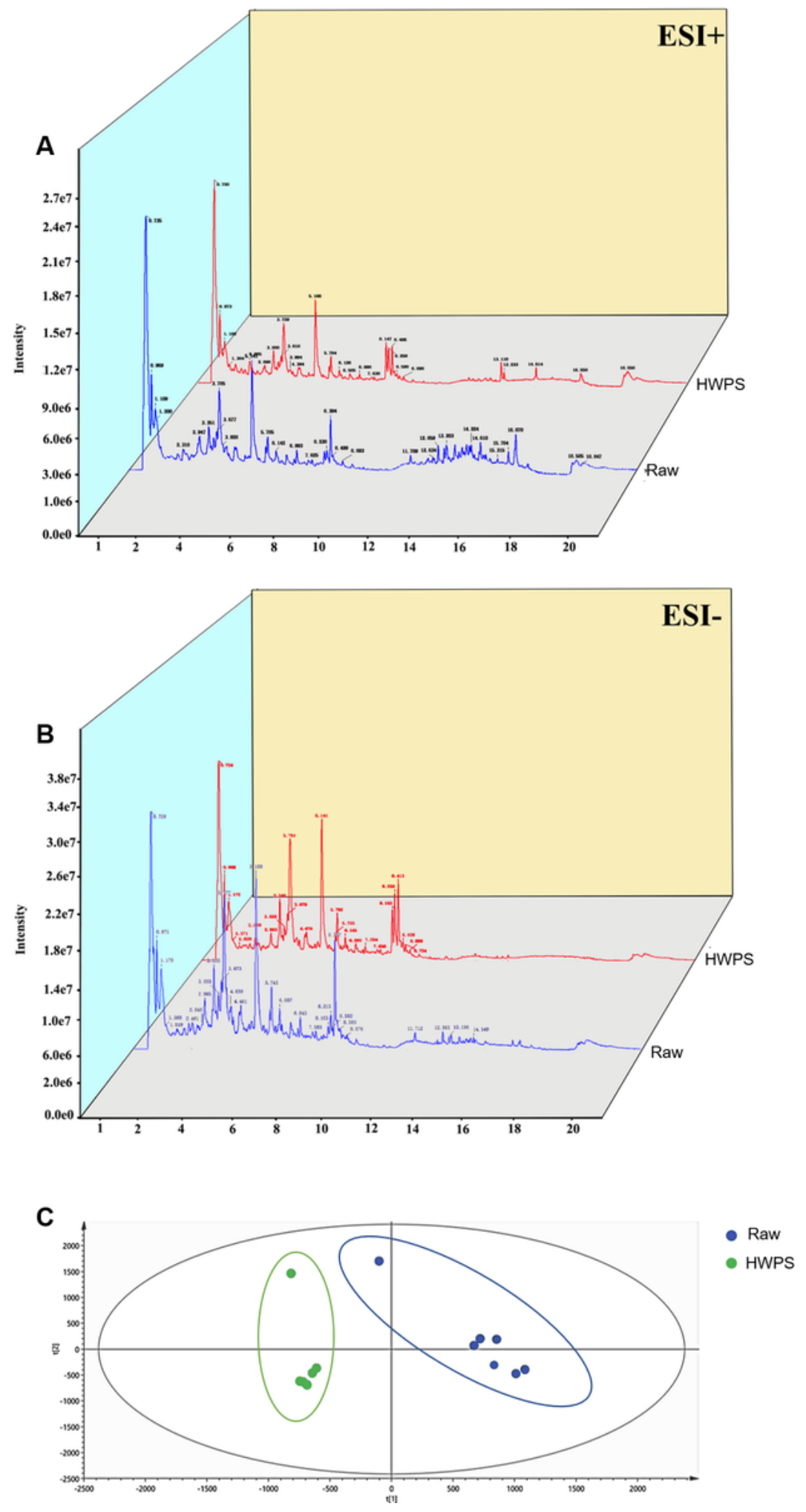

Figure 5

Ingredient identification of $\mathrm{C}$. officinalis processed with HPWS. A. Raw sample positive and negative ion pattern diagram. B. The product's positive and negative ion pattern is illustrated. C. PLS-DA score plot.

\section{Supplementary Files}


This is a list of supplementary files associated with this preprint. Click to download.

- GraphicalAbstract20211029.tif

- Table1.pdf

- Supplementary.pdf 